\title{
Organized Hemopericardium Mimicking Malignant Tumor: A Case of Spontaneous Pericardial Hematoma In a 23-Year-Old Young Male
}

\author{
Wenda Wang, Qi Miao \\ The Department of Cardiac Surgery, Peking Union Medical College Hospital, Chinese Academy of Medical Sciences, \\ Peking Union Medical College, Beijing, China
}

\section{ABSTRACT}

Spontaneous pericardial hematoma is rare, and the diagnosis of spontaneous organized hemopericardium is difficult in some cases. The present study reports a case of a 23-yearold man admitted to our hospital for dyspnea on Sept. 30, 2017. The patient experienced shortness of breath 10 days before admission and went to a local hospital. The chest noncontrast CT and Echo revealed effusion of pericardium, right thoracic cavity and abdominal cavity. The patient went to our hospital for further treatment, and there was no abnormality in the laboratory tests of infection, tumor, or immune disease. The patient received pericardiocentesis for cardiac tamponade. A re-examination of Echo after pericardiocentesis revealed a pericardial mass. The enhanced chest CT and 18 F-FDG PET/CT could not rule out the risk of a malignant tumor. The resection of pericardial mass was performed under cardiopulmonary bypass with cardioplegia. The pathology was organized hematoma with widely hemorrhage and necrosis, fibrous tissue proliferation, myofibroblast proliferation, and granulation tissue formation. The patient was discharged from our hospital without recurrence of pericardial effusion or the symptom of dyspnea. This case suggests that spontaneous organized hemopericardium is difficult to be distinguished from malignant tumor, and a surgical resection can help with diagnosis and symptomatic relief.

\section{INTRODUCTION}

Spontaneous pericardial hematoma lacking a trauma or surgical history is relatively rare [Tokue 2013], and spontaneous organized hematoma is difficult to be distinguished from malignant tumor [Tokue 2013; Alter 2013]. We experienced a rare case of spontaneous organized hemopericardium in a young male which mimicked pericardial tumor.

\section{CASE REPORT}

A 23-year-old male, who had felt suffocated for 10 days, came to our hospital on Sept. 30, 2017. The patient initially

Received April 24, 2018; accepted August 6, 2018.

Correspondence: Qi Miao, Department of Cardiac Surgery, Peking Union Medical College Hospital, Chinese Academy of Medical Sciences, Peking Union Medical College, Beijing 100730, China. Telephone and fax: 86-69152813 (e-mail:miaoqipumc@botmail.com). experienced shortness of breath after fast walking 10 days before admission. The dyspnea got aggravated and was affected by lying down one week later. Therefore, the patient went to a local hospital for examination. Chest and abdominal non-contrast computerized tomography (CT) showed effusion of pericardium, right thoracic cavity and abdominal cavity. Echocardiography (Echo) presented massive pericardial effusion with left ventricular diastolic dysfunction. However, the examination of Echo did not reveal any intrapericardial mass clearly.

The patient came to emergency department of our hospital for further treatment on Sept. 30. Upon examination, blood pressure was $94 / 72 \mathrm{mmHg}$ and heart rate was $122 \mathrm{bpm}$. Laboratory studies revealed a slight higher level of white blood cell (WBC) $\left(10.27 \times 10^{9} / \mathrm{L}, \mathrm{NEUT} \% 79.4 \%\right)$ with a slight lower hemoglobin level $(118 \mathrm{~g} / \mathrm{L})$. Coagulation function revealed almost normal PT (14s, normal range 10.4-12.6s) and APTT (32.1s, normal range 22.7-31.8s). There was mild increase in the levels of two tumor markers, including CA125 (62.4U/ $\mathrm{mL}$, normal range $0-35.0 \mathrm{U} / \mathrm{mL}$ ) and TPS $(129.48 \mathrm{U} / \mathrm{L}$, normal range $0-80 \mathrm{U} / \mathrm{mL})$, while the others were normal (CA242, SCCAg, PSA, AFP, CEA, CA19-9, CA15-3, NSE, and Cyfra211). CRP was $19.50 \mathrm{mg} / \mathrm{L}$ (normal range $0-3 \mathrm{mg} / \mathrm{L}$ ) and ESR was $5 \mathrm{~mm} / \mathrm{h}$ (normal range $0-15 \mathrm{~mm} / \mathrm{h}$ ). There was no abnormality of infection indexes, including procalcitonin (PCT), T.SPOT-TB, and blood culture of bacteria, fungi and mycobacteria. The immune indexes were also normal.

The diagnosis of cardiac tamponade was established and a therapeutic pericardiocentesis was performed on the day of admission. A total of $1500 \mathrm{~mL}$ of hemorrhagic fluid was removed. Cytology revealed a WBC count was 3832 cells $/ \mathrm{mm}^{3}$, with a differential cell count of polymorphonuclear cells of $69.5 \%$. The Gram stain and acid-fast stain were negative. No positive findings were detected in the culture of bacteria and fungi. For cytopathology, plenty of mesothelial cells were present, but no malignant cells were found with an immunohistochemical result of Calretinin (+), D2-40 (+), CK5/6 (partial +), Desmin (+), BerEP4 (-), Napsin A (-), TTF-1 (-), P63 (-), P40 (-).

A re-examination of echocardiography on the 20th day after initial of symptoms revealed a large mass $(3 \mathrm{~cm} \times 6 \mathrm{~cm})$ with middle echogenicity located beside left lateral and anterior ventricular wall. An 18F-FDG PET/CT was performed, revealing uptake in the peripheral rim of the intrapericardial mass above the left ventricle, and the maximum standardized uptake value (SUV) was 3.9. PET/CT could not rule out the risk of a malignant lesion. An enhanced chest CT was also performed, and it revealed a mass $(40.4 \mathrm{~mm} \times 49.6 \mathrm{~mm})$ with mixed density (Figure 1). There was a clear boundary 

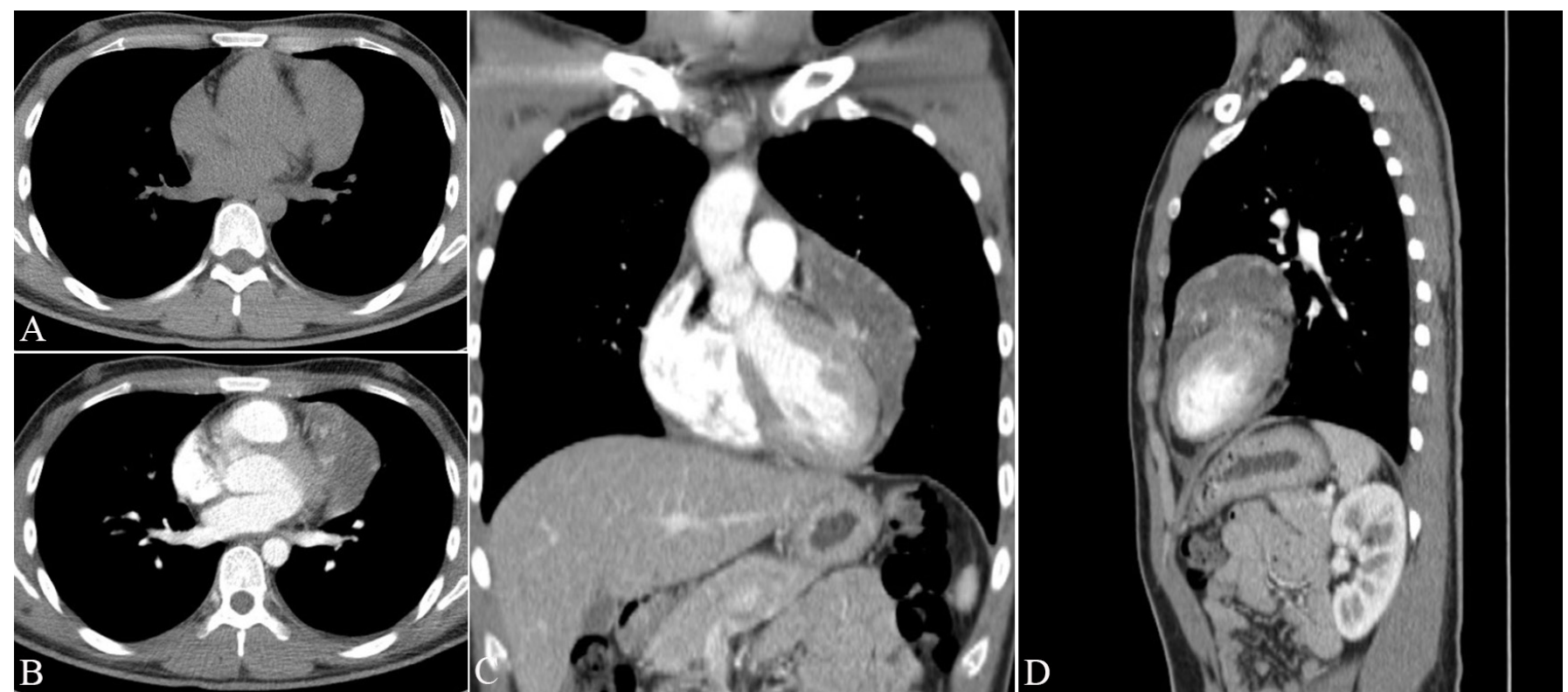

Figure 1. CT scan showing a pericardial mass lacking calcification with enhancement. (A-B) Axial view of plain scan, (C-D) axial view of enhancement scan, and (E-F) multiplanar reconstruction view.
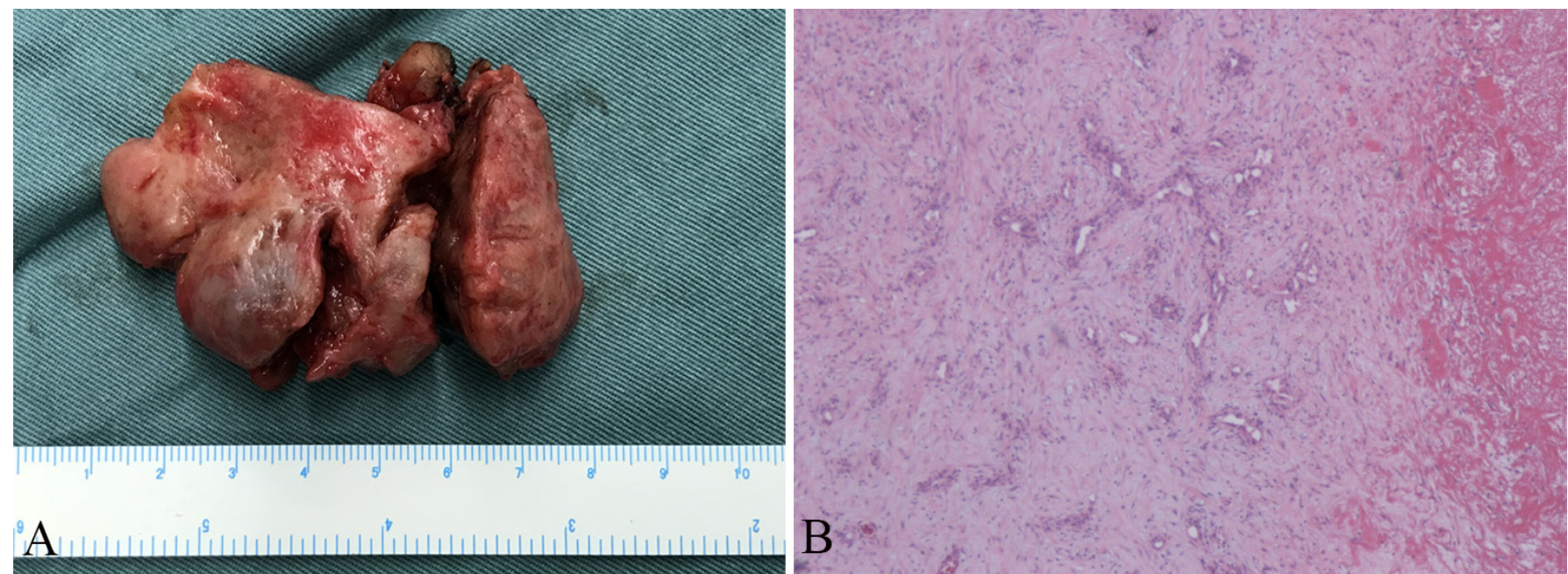

${ }^{\circ} \mathrm{A}$

Figure 2. The gross and microscopic pathology of the pericardial mass. (A) The cauliflower-like mass attaching to the surface of left ventricle, and (B) the HE staining showing the pathology of organized hematoma.

between the lesion and left ventricle. It lacked calcification and showed enhancement of edge and internal division.

We performed surgical resection under extracorporeal circulation on the 50th day after initial symptoms and after securing informed consent. The pericardium was widely adhered to the heart, especially on the left side. The adhesion was detached carefully and we found a $4 \mathrm{~cm} \times 6 \mathrm{~cm}$ cauliflower-like mass attaching to the surface of the left ventricle, locating between the left anterior descending artery and left circumflex artery. The mass was smooth and suspectedly encapsulated. Complete removal of the mass was successful with excising partial left atrial appendage (Figure 2a), and the residual left atrial appendage was sutured.

A fast-frozen pathology during operation revealed that the mass might be a spindle cell tumor with hemorrhage and degeneration. However, the final pathology revealed there was wide hemorrhaging and necrosis, fibrous tissue proliferation, myofibroblast proliferation and granulation tissue formation (Figure 2b). The immunohistochemical results revealed AE1/AE3(partial +), CD34(-), CD31(-), Desmin(focal +), Ki-67(index 5\%), Myoglobin(-), SMA(+), S-100(-). The final conclusion was organized hematoma. The patient was discharged after recovery without pericardial effusion or any discomfort on the ninth day after operation. The patient had no complaints of dyspnea when he came for re-examination at six months after operation, and the echocardiography revealed no relapse of pericardial mass or effusion. 


\section{DISCUSSION}

The final diagnosis was organized hematoma. Reviewing the progression of disease, we found that the patient's first clinical manifestation was pericardial effusion with dyspnea. No pericardial mass was presented on the chest CT plain scan or Echo at 10 days after onset of symptoms probably because of the cover of bloody effusion. At 12 days, the patient received pericardial drainage. A re-examination of Echo after pericardiocentesis at 21 days revealed the pericardial mass. Then, the resection of pericardial mass was performed and the pathology was organized hematoma.

Hemorrhagic effusion of pericardium is more often found in population with cardiac surgery, trauma, or coagulation disorders. When trauma is absent, a hemorrhagic effusion of pericardium strongly suggests a malignant lesion. Some cases have been found to have rare etiologies [Cordes 1999]. Ueyama et al reported a case of intrapericardial hematoma due to coronary to pulmonary artery fistulas [Ueyama 2015], and Nakanishi et al published a case of spontaneous pericardial hematoma caused by amyloid [Nakanishi 2009].

Despite all this, hemorrhage could occur after slight trauma with no awareness or even spontaneously. The mechanism of spontaneous hematoma is incompletely understood, but people have found that during the process, the irritation releases vasoactive substances and induces capsule formation, while repeated inflammation will lead to effusion and new bleeding from damaged microvessels beneath the capsule [Sughiura 2006]. Our patient had no surgical or trauma history, and the coagulation function was normal. There was no malignant tumor, which was proven by final pathology. Therefore, the spontaneous pericardial hematoma might be established.

Most hematomas can resolve spontaneously, but some cannot and will become organized. The organized hemopericardium frequently lead to constrictive pericarditis causing heart failure [Velibey 2014]. There are several cases of organized pericardial hematoma reported after several years after trauma or surgery [Velibey 2014; Chamsi-Pasha 2011], but the spontaneous cases are rare. Our report presented an interesting case which had onset symptoms of pericardial effusion and cardiac tamponade. It was hard to confirm the time of organized hematoma formation because the patient lacked a history of surgery or trauma.

The patient's pericardial mass was similar with a malignant tumor, especially in the absence of surgery or trauma history. We could not identify it from a malignant lesion no matter according to imaging characteristics or gross specimen. Even so, imaging assessment should always be an indispensable tool. CT scan can detect the high attenuation of initial blood, and it would decrease as time progresses. Then, CT scan can detect fibrosis and calcification with high sensitivity. Hematomas will not enhance with iodinated contrast [Alter 2013]. However, our patient's CT images revealed heterogeneous enhancement but no calcification, and this could cause confusion for the diagnosis. On MRI, the appearance of a pericardial hematoma also changes with the age of it. A fresh hematoma shows high signal intensity on T1WI and T2WI, but a chronic organized hematoma reveals low signal intensity [Maksimović 2006]. A tumor on MRI may present high signal intensity on T2WI with contrast enhancement. Although our patient did not perform an MRI, it may be also difficult to differentiate pericardial hematoma from tumor by MRI for the signal intensity of hematoma is variable during the process from acute stage to chronic stage. PET/CT is also limited by the fact that FDG is taken up not only by tumor cells but also by macrophages or tissue with granulation and inflammation [Tokue 2013], and an organized hematoma can mimic a malignant tumor on PET/CT. So, it is hard to distinguish them from each other in this case.

Because organized pericardial hematoma may lead to constrictive pericarditis in some cases, a surgical resection may be appropriate. The procedure can help to establish the final diagnosis, and also can prevent or remit pericardial tamponade and constriction improving cardiac function [Velibey 2014].

\section{CONCLUSION}

Spontaneous pericardial hematoma is rare, and it may be difficult to distinguish the organized hematoma from malignant tumor according to imaging examinations. A surgical excision can help to establish the diagnosis and resolve the complications.

\section{REFERENCES}

Alter P, Figiel JH, Rupp TP, Bachmann GF, Maisch B, et al. 2013. MR, CT, and PET imaging in pericardial disease. Heart Fail Rev. 18(3):289-306.

Chamsi-Pasha MA, Anwar AM, Nosir YF, Ajam A, Abukhudair W, et al. 2011. Organized intrapericardial haematoma: a rare cause of heart failure after 2 years of trauma. Eur J Echocardiogr. 12(1):E1.

Cordes D, DeGroff C, Shaffer EM. 1999. Spontaneous pericardial hematoma in an infant. Pediatr Cardiol. 20(5):380-1.

Maksimović R, Dill T, Seferović PM, Ristić AD, Alter P, et al. 2006. Magnetic resonance imaging in pericardial diseases. Indications and diagnostic value. Herz. 31(7):708-14.

Nakanishi N, Sawada T, Sato R, Yanishi K, Akakabe Y, et al. 2009. Spontaneous pericardial hematoma with familial amyloid polyneuropathy. Amyloid. 16(4):221-5.

Sughiura T, Nishida H, Ishitoya H, Tomizawa Y, Saito S, et al. 2006. Chronic expanding intrapericardial hematoma after pericardial paracentesis. J Card Surg. 21(5):491-3.

Tokue H, Tokue A, Okauchi K, Tsushima Y. 2013. 2-[18F]fluoro-2-deoxy-D-glucose (FDG) positronemission tomography (PET) findings of chronic expanding intrapericardial hematoma: a potential interpretive pitfall that mimics a malignant tumor. J Cardiothorac Surg. 8:13.

Ueyama K, Morishima M, Iida J. 2015. First report of chronic expanding intrapericardial hematoma caused by coronary to pulmonary artery fistulas. Ann Thorac Surg. 99(3):1084.

Velibey Y, Sahin S, Altay S, Bakshaliyev N, Tusun E, et al. 2014. A rare cause of congestive heart failure after seven years of open heart surgery: Organized intrapericardial hematoma. Anadolu Kardiyol Derg. 14(2):205-8. 